\title{
Pure Salivatory Seizures Secondary to a Subtle Malformation of the Right Parietal Cortex
}

\author{
Fábio A. Nascimento ${ }^{*}$, Danah Aljaafari1,2, Murilo de Souza Meneses ${ }^{3}$, \\ Samanta Fabrício Blattes da Rocha ${ }^{3}$, Cristiane Simão ${ }^{3}$, Pedro A. Kowacs ${ }^{3}$ \\ ${ }^{1}$ Division of Neurology, Toronto Western Hospital, University of Toronto, Toronto, Canada \\ ${ }^{2}$ Deparment of Neurology, King Fahad Hospital of University, University of Dammam, Dammam, Saudi Arabia \\ ${ }^{3}$ Neurological Institute of Curitiba (INC), Curitiba, Brazil \\ Email: *Nascimento.Fabio.A@gmail.com, Danah.Aljaafari@uhn.ca, Mmeneses@onda.com.br, \\ Samanta@habilittare.com, Cris_cris_@hotmail.com,PKowacs@gmail.com
}

Received 5 January 2016; accepted 7 March 2016; published 10 March 2016

Copyright (C) 2016 by authors and Scientific Research Publishing Inc.

This work is licensed under the Creative Commons Attribution International License (CC BY).

http://creativecommons.org/licenses/by/4.0/

(c) $\underset{\mathrm{EY}}{\mathrm{O}}$ Open Access

\begin{abstract}
Background: Salivatory seizures are a singularly rare condition, which can occur both in idiopathic and symptomatic epilepsies. Objectives: To describe and discuss the case of an adolescent patient with sleep-triggered "pure" salivatory seizures associated with a subtle cortical malformation of the right parietal cortex. Case report: Herein, we report a 17-year-old female who started to present salivatory paroxysms, which occasionally secondarily generalized, shortly after falling asleep, at the age of eight years. Video-electroencephalographic monitoring with scalp electrodes failed to show any epileptiform activity during the several recorded clinical events. Brain MRI and curvilinear reconstruction revealed, in the three orthogonal planes, a subtle cortical thickening, limited to a single gyrus in the right parietal cortex, suggestive of a focal cortical malformation. After antiepileptic drug therapy was optimized, the patient became seizure-free. Conclusion: An epilepsy diagnosis should be pursued in patients presenting isolated, paroxysmal hypersalivation, despite possible negative scalp EEG studies.
\end{abstract}

\section{Keywords}

Salivatory Seizures, Malformation of Cortical Development, Hypersalivation, Sialorrhea

\section{Introduction}

Salivatory seizures are a rare type of focal seizures without impairment of consciousness or awareness. Saliva-

"Corresponding author.

How to cite this paper: Nascimento, F.A., Aljaafari, D., de Souza Meneses, M., da Rocha, S.F.B., Simão, C. and Kowacs, P.A. (2016) Pure Salivatory Seizures Secondary to a Subtle Malformation of the Right Parietal Cortex. Neuroscience \& Medicine, 7, 10-14. http://dx.doi.org/10.4236/nm.2016.71002 
tion as an autonomic manifestation of Rolandic cortex seizure activity was first described by Penfield and Jasper, 1954 [1]. Focal or spreading electrical activity, involving Rolandic topography, is known to generate salivation, as well as sensory and motor orofacial features. Nonetheless, isolated salivatory epileptic phenomena are singularly rare. The first reported case of salivatory seizures was described in 1954, by Penfield and Jasper [1]. This patient had a posttraumatic cyst in the right postcentral gyrus, which led to focal epilepsy manifesting as increased salivation. Notably, after cyst resection, seizure freedom was achieved. Subsequently, further cases of salivatory seizures in association with variable degrees of oromotor dysfunction and other select clinical features were published [2]-[9]. Recognition of increased salivation as seizures is of paramount importance, mainly because these paroxysms have a fairly good response to clinical and/or surgical treatments. Herein, we report an otherwise healthy 17-year-old female who presented salivatory seizures since the age of eight years.

\section{Case Report}

The patient is a 17-year-old female adolescent who started to present seizures at the age of eight years. Her spells were characterized by an initial tingling sensation inside her mouth followed by trismus and sialorrhea, which then led to voluntary spitting, without impairment of consciousness or awareness. These events were nocturnal, occurring soon after falling asleep, and lasted approximately half a minute. Regarding their frequency, it was erratic thus hard to determine. Occasionally these seizures secondarily generalized. Physical, neurological and psychiatric examinations were normal. There was no evidence neither of gastrointestinal nor otorrhynolaringological disease. At presentation at our centre, she was using carbamazepine $200 \mathrm{mg}$ three times per day (maximal tolerated dose), without seizure control. Previously, she had tried phenobarbital, valproate, phenytoin, primidone, topiramate and clobazam - all of which had failed to control her epilepsy. A video-EEG analysis, performed at our hospital, registered various clinical seizures but failed to record any epileptic activity. Brain MRI and curvilinear reconstruction revealed, in the three orthogonal planes, a subtle cortical thickening, limited to a single gyrus at the right parietal cortex, suggestive of a focal cortical malformation (Figure 1). We then decided to optimize her medical treatment by adding lamotrigine to her antiepileptic drug regimen, which was titrated up to $75 \mathrm{mg}$ three times per day. Subsequently, the patient became seizure-free. Presently, at 10-year follow-up, she still uses carbamazepine (at 200/200/400 mg) and lamotrigine (at 100/100/200 mg) and continues to be seizure-free except for breakthrough seizures in the setting of missed medications.

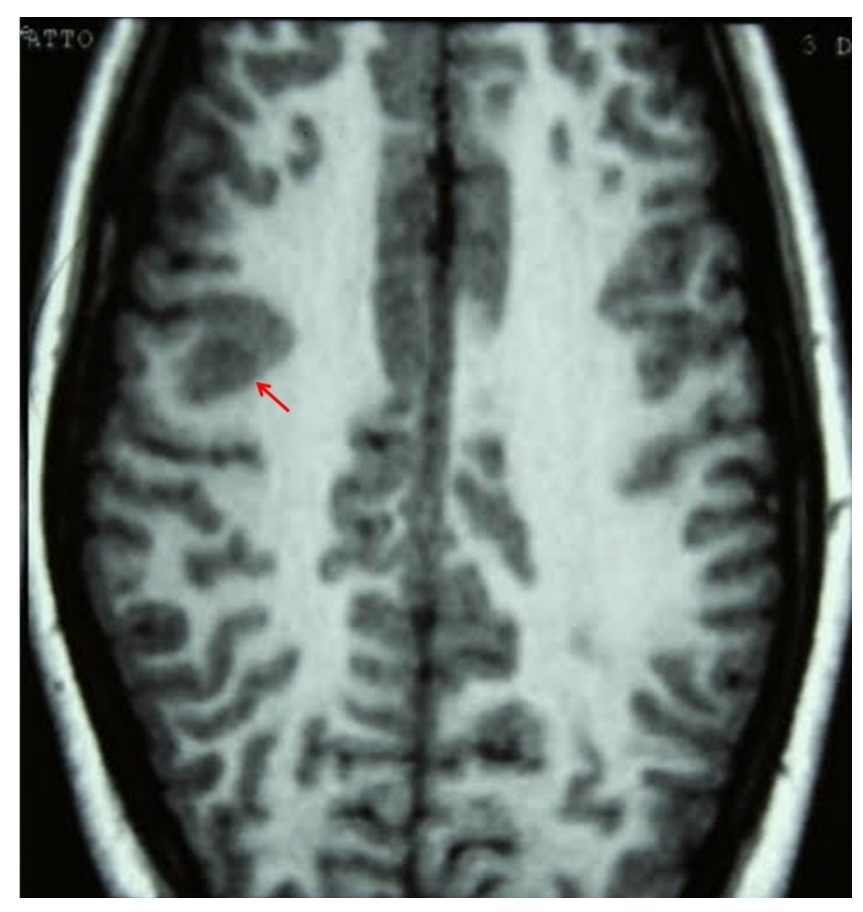

Figure 1. Brain MRI revealed a subtle cortical malformation in the right parietal topography. 


\section{Discussion}

Autonomic symptoms are often associated with seizures, typically in combination with other epileptic manifestations. Generated by activation or inhibition of portions of the central autonomic network, autonomic disturbances depend on specific cerebral areas involved in seizure onset or propagation. Seizures comprising exclusively autonomic symptoms, however, are rarely reported [10].

"Pure” salivatory seizures were primarily recognized by Penfield and Jasper, in 1954 [1]. These authors reported a patient with abundant drooling paroxysms, which were associated with continuous focal seizures. Ictal hypersalivation happened mostly during the day, and was conscientiously perceived by this patient. Seizure freedom was achieved after resection of a traumatic cyst located in the postcentral gyrus. It was not until 10 years after that two additional cases of salivatory seizures were reported [9].

Hypersalivation, usually as an associated ictal symptom, has been repeatedly associated with several epilepsy syndromes, such as Rolandic epilepsy, Panayiotopoulos syndrome, temporal lobe epilepsy, among others [10]. In terms of localization, hypersalivation has been associated with diverse topography - rolandic area, fronto-or bital cortex and cingulate gyrus, insula, operculum, and mesial temporal structures [4].

Specifically focusing on Rolandic epilepsy (or benign childhood epilepsy with centro-temporal spikes, BCECTS), there have been quite a few reports of this population presenting temporary oromotor symptoms as epileptic manifestations. These include not only increased salivation but also speech arrest, anarthria, dysphagia, lingual dyspraxia, etc. [3] [7] [11]. In this context, Roulet et al. reported a BCECTS patient who experienced prolonged, intermittent drooling and lingual dyspraxia. Ictal EEG studies showed paroxysmal discharges arising from both Rolandic areas. The fluctuant course of these clinical phenomena and correlation with the intensity and localization of the paroxysmal discharges on EEG supported the hypothesis of an epileptic dysfunction located in the lower Rolandic fissure-in agreement with Penfield and Jasper's observations on cortical representation of salivation [3].

Colomaria et al. reported a patient diagnosed with BCECTS with seizures characterized by speech arrest, drooling, and swallowing difficulties. These events, when monitored by continuous EEG, were found to be consistent with status epilepticus (SE). Interestingly, electroencephalographic records showed additional patterns of continuous spike-wave during slow wave sleep. Seizures were controlled with benzodiazepines [6].

Fejerman et al. studied 26 children with BCECTS who shared atypical evolutions-including SE and language, cognitive or behavioural impairments. Seven of these subjects presented SE. Five of them had status of unilateral, facial motor seizures, anarthria, and sialorrhea; two had prolonged status of anarthria and sialorrhea. This study concluded that the oromotor deficits (including hypersalivation) were due to discharges in the lower rolandic areas [8].

In a longitudinal assessment of a patient with BCECTS and fluctuating oromotor deficits (drooling, dysarthria, and dysphagia), de Saint-Martin et al. tried to identify if these phenomena were actually ictal symptoms (as in the previously described SE of BCECTS) or secondary to interictal epileptiform activity. These researchers hypothesized that, in BCECTS, oromotor seizures might be the result of either ictal or interictal epileptiform activity in Rolandic topography [12]. Evidence that corroborated the aforementioned hypothesis came from a case series conducted by Kramer et al. Five patients with BCECTS and intermittent dysarthria and drooling were assessed. Ictal EEG studies in four of them showed electroencephalographic correlation, as continuous epileptiform discharges. The remaining patient, however, did not have any alteration in EEG during the clinical events. In contrary, this last patient had only an increase of the frequency of interictal discharges. Thus, the authors concluded that the deficit on the latter child could have been due to an increased interictal epileptiform activity, as part of an inhibitory mechanism affecting specific cerebral autonomic functions [5].

Transient oromotor symptoms, as mentioned above, have also been associated with temporal lobe epilepsy. Twelve patients with a history of ictal spitting were studied by Kellinghaus et al. In 11 of the 12 the epileptogenic zone was located to the temporal lobe. Based on EEG studies, six of them had a seizure onset clearly lateralized to the right, nondominant hemisphere, maximally over the temporal region [13].

It has recently been reported a case of left mesial temporal lobe epilepsy due to hippocampal sclerosis, with concordant MRI and EEG, presenting with a seizure semiology characterized by initial upper abdominal discomfort followed by massive salivation. This patient became seizure-free after left temporal lobectomy. The authors attributed the ictal salivation to the activation of frontal limbic system triggered by hippocampal abnormal firing during seizure onset [4]. According to this study, the prevailing explanation for sialorrhea accompanying 
temporal lobe epilepsy consists of spreading of ictal discharges from the mesial temporal structures.

Increased ictal salivation has also been linked to lesional focal epilepsy, for instance in the opercular cortex. A 26-year-old patient with seizures characterized by throat discomfort and hypersalivation was found to have an atrophic lesion around the peri-Sylvian area. Although scalp EEG failed to demonstrate robust, localizable epileptiform activity, invasive EEG showed seizures arising from the left frontal operculum. After lesionectomy, this patient became seizure-free.

Returning to our patient, although several clinical seizures were recorded during video-EEG monitoring with scalp electrodes, epileptiform activity could not be demonstrated. Nevertheless, due to the fact that the MRI study of this patient showed a subtle cortical thickening of a single parietal gyrus, in addition to significant clinical response to antiepileptic drug therapy, we believe that the events experienced by our patient is indeed of epileptic origin. In terms of electroencephalographic correlation, we believe that scalp EEG failed to show epileptiform activity due to the presence of a tangential dipole effect.

In fact, there has been a report of a pediatric patient with epilepsy presenting with paroxysms of drooling, dysarthria, and dysphagia. Ictal EEG, similarly to our case, failed to confirm epileptogenicity; further, there were no structural abnormalities present in neuroimaging studies. Interestingly, this patient became free of seizures after initiated on antiepileptic drug therapy. The authors hypothesized that the seizures originated from "silent" cortical areas [14].

By reporting this case, we highlight the importance of considering a diagnosis of epilepsy in patients with paroxysmal hypersalivation, despite negative scalp EEG studies. Based on our case, as well as on the patients discussed above, an impressive response to clinical and/or surgical treatment can be missed if epilepsy is not considered in these particular situations.

\section{Disclosure}

This study was not funded. There is no conflict of interest. All authors report no disclosures.

\section{Funding}

This work was neither presented at any meeting nor funded or supported by any sort of funding.

\section{References}

[1] Penfield, W. and Jasper, H. (1954) Epilepsy and the Functional Anatomy of the Human Brain. Little, Brown, Boston, 363-365.

[2] Satow, T., Ikeda, A., Hayashi, N., et al. (2004) Surgical Treatment of Seizures from the Peri-Sylvian Area by Perinatal Insult: A Case Report of Ictal Hypersalivation. Acta Neurochir (Wien), 146, 1021-1025. http://dx.doi.org/10.1007/s00701-004-0311-7

[3] Roulet, E., Deonna, T. and Despland, P.A. (1989) Prolonged Intermittent Drooling and Oromotor Dyspraxia in Benign Childhood Epilepsy with Centrotemporal Spikes. Epilepsia, 30, 564-568. http://dx.doi.org/10.1111/j.1528-1157.1989.tb05472.x

[4] Morita, M., Kurita, A., Inoue, K., Shimizu, H., Mizutani, T. and Arai, N. (2001) A Case with Left Mesial Temporal Lobe Epilepsy Characterized by Abnormal Massive Salivation. Rinsho Shinkeigaku, 41, 809-812.

[5] Kramer, U., Ben-Zeev, B., Harel, S. and Kivity, S. (2001) Transient Oromotor Deficits in Children with Benign Childhood Epilepsy with Central Temporal Spikes. Epilepsia, 42, 616-620. http://dx.doi.org/10.1046/j.1528-1157.2001.28700.x

[6] Colamaria, V., Sgro, V., Caraballo, R., et al. (1991) Status Epilepticus in Benign Rolandic Epilepsy Manifesting as Anterior Operculum Syndrome. Epilepsia, 32, 329-334. http://dx.doi.org/10.1111/j.1528-1157.1991.tb04659.x

[7] Boulloche, J., Husson, A., Le Luyer, B. and Le Roux, P. (1990) Dysphagia, Speech Disorders and Centrotemporal Spikes-Waves. Archives Françaises de Pédiatrie, 47, 115-117.

[8] Fejerman, N., Caraballo, R. and Tenembaum, S.N. (2000) Atypical Evolutions of Benign Localization-Related Epilepsies in Children: Are They Predictable? Epilepsia, 41, 380-390. http://dx.doi.org/10.1111/j.1528-1157.2000.tb00177.x

[9] Torii, H., Hayashi, M. and Shimazono, Y. (1963) Two Cases of Epilepsy with Salivary Seizures. No To Shinkei, 15, 783-792.

[10] Panayiotopoulos, C.P., Michael, M., Sanders, S., Valeta, T. and Koutroumadis, M. (2008) Benigh Childhood Focal Epilepsies: Assessment of Established and Newly Recognized Syndromes. Brain, 131, 2264-2286. 
http://dx.doi.org/10.1093/brain/awn162

[11] Fejerman, N. and Di Blasi, A.M. (1987) Status Epilepticus of Benign Partial Epilepsies in Children: Report of Two Cases. Epilepsia, 28, 351-355. http://dx.doi.org/10.1111/j.1528-1157.1987.tb03656.x

[12] de Saint-Martin, A., Petiau, C., Massa, R., et al. (1999) Idiopathic Rolandic Epilepsy with “Interictal” Facial Myoclonia and Oromotor Deficit: A Longitudinal EEG and PET Study. Epilepsia, 40, 614-620. http://dx.doi.org/10.1111/j.1528-1157.1999.tb05564.x

[13] Kellinghaus, C., Loddenkemper, T. and Kotagal, P. (2003) Ictal Spitting: Clinical and Electroencephalographic Features. Epilepsia, 44, 1064-1069. http://dx.doi.org/10.1046/j.1528-1157.2003.67302.x

[14] Gayatri, N.A., Hughes, M.I., Clarke, M.A. and Martland, T.R. (2002) Epilepsy with Reversible Bulbar Dysfunction. Developmental Medicine \& Child Neurology, 44, 770-772. http://dx.doi.org/10.1111/j.1469-8749.2002.tb00284.x 